INDO GLOBAL JOURNAL OF

PHARMACEUTICAL SCIENCES

ISSN 2249- 1023

\title{
Insight into the Gut-Microbiome of Indian Indigenous Chicken
}

\author{
Sharad Saxena ${ }^{1}$, V K Saxena ${ }^{2}$, Simmi Tomar ${ }^{2}$, Vibha Rani ${ }^{1 *}$ \\ ${ }^{1}$ Department of Biotechnology, Jaypee Institute of Information Technology, A-10, Sector-62, Noida - 201307, UP, India \\ ${ }^{2}$ Division of Avian Genetics and Breeding, Central Avian Research Institute, Izatnagar, Bareilly 243122, UP, India
}

Address for Correspondance: Vibha Rani, vibha.rani@jiit.ac.in

Keywords

Chicken Gut-

Microbiota; $16 \mathrm{~S}$

rRNA; Probiotics;

MG-RAST.
ABSTRACT: Indian indigenous chickens are known to be hardy and highly adapted to the native environment as compared to the commercial poultry strains. The gut micobiota is known to play an important role in nutrient utilization, growth, and well-being of these birds. The variations in gut microbiota composition may be associated with adaptability traits to the indigenous birds. To identify uncharacterized microbial strains from gut-microbiome of indigenous birds, we explored microbial composition of different gastrointestinal segments viz. Crop, Jejunum, Ileum and Cecum. 16S rRNA sequencing targeting V3, V4, and V4-V6 hypervariable regions was performed using next-generation sequencing. A total of 1,975,492 paired end raw reads were obtained from metagenomic DNA of all four segments. The metagenomic datasets were annotated to both M5NR and M5RNA databases under MG-RAST server. Alpha diversity analysis revealed that cecum has the highest bacterial diversity whereas ileum has the least. Fermicutes, Bacteroidetes and Proteobacteria were the dominant taxa with variable distribution between segments. Analysis at higher taxonomic level revealed that bacterial composition for each segment was specific. We detected lignocellulose degrading bacterial species like Clostridium cellulolyticum, Clostridium phytofermentans and Acetivibrio cellulolyticus. Also, for the first time we report the presence of Lactobacillus species such as L. antri, L. mucosae in chicken gut which are phylogenetic relatives of known probiotic species. These microbes might be serving the birds to adapt in native environment and could be employed to manipulate commercial chicken microbiota in order to improve its performance.C 2016 iGlobal Research and Publishing Foundation. All rights reserved.

Conference Proceedings: International Conference on Advances in Plant and Microbial Biotechnology (PMB2017); JIIT, Noida: February 02-04, 2017

Indo Global Journal of Pharmaceutical Sciences( ISSN 22491023 ; CODEN- IGJPAI; NLM ID: 101610675) indexed and abstracted in EMBASE(Elsevier), SCIRUS(Elsevier),CABI, CAB Abstracts, Chemical Abstract Services(CAS), American Chemical Society(ACS), Index Copernicus, EBSCO, DOAJ, Google Scholar and many more. For further details, visit http://iglobaljournal.com 